\title{
Oscillation Criteria of Second-Order Nonlinear Differential Equations with Variable Coefficients
}

\author{
Ambarka A. Salhin, Ummul Khair Salma Din, \\ Rokiah Rozita Ahmad, and Mohd Salmi Md Noorani
}

School of Mathematical Sciences, Faculty of Science and Technology, Universiti Kebangsaan Malaysia (UKM), 43600 Bangi, Selangor, Malaysia

Correspondence should be addressed to Ambarka A. Salhin; amb80ark@yahoo.com

Received 31 August 2013; Revised 27 December 2013; Accepted 31 December 2013; Published 18 February 2014

Academic Editor: M. De la Sen

Copyright (C) 2014 Ambarka A. Salhin et al. This is an open access article distributed under the Creative Commons Attribution License, which permits unrestricted use, distribution, and reproduction in any medium, provided the original work is properly cited.

Some new oscillation criteria are given for second-order nonlinear differential equations with variable coefficients. Our results generalize and extend some of the well-known results in the literatures. Some examples are considered to illustrate the main results.

\section{Introduction}

Since many problems in physics, chemically reacting systems, celestial mechanics, and others fields are modeled by second-order nonlinear differential equations, the oscillatory and asymptotic behaviors of solutions of such differential equations have been investigated by many authors [1-9]. Investigation of nonlinear differential equation in this paper is motivated by [1], where some of the conditions required in the theorems contain the unknown solution $x(t)$. It seems that any verification of such conditions is questionable.

In this paper, we consider the oscillation of second-order nonlinear forced differential equation

$$
\begin{array}{r}
\left(r(t) \psi(x(t)) f\left(x^{\prime}(t)\right)\right)^{\prime}+q(t) g(x(t)) \\
=H\left(t, x^{\prime}(t), x(t)\right), \quad t \in\left[t_{0}, \infty\right),
\end{array}
$$

where $r, q \in C\left(\left[t_{0}, \infty\right), \mathbb{R}\right)$ and $f, \psi, g \in C(\mathbb{R}, \mathbb{R})$ and $H$ is a continuous function on $\left[t_{0}, \infty\right) \times \mathbb{R}^{2}$.

Consider the following with respect to (1):

$\left(\mathrm{A}_{1}\right) r(t)>0, t \geq 0$;

$\left(\mathrm{A}_{2}\right) \psi(x) \in C(\mathbb{R}), \psi(x)>0$ for all $x$;

$\left(\mathrm{A}_{3}\right) x g(x)>0, g \in C^{1}(\mathbb{R})$ for $x \neq 0$;

$\left(\mathrm{A}_{4}\right) H(t, y, x) / g(x) \leq p(t)$ for all $t \in\left[t_{0}, \infty\right) ; x, y \in \mathbb{R}$ and $x \neq 0$.
We say that a nonzero function $x:\left[t_{0}, t_{1}\right) \rightarrow(-\infty, \infty), t_{1}>$ $t_{0}$ is called a nontrivial solution of (1) if $x(t)$ satisfies (1) for all $t \in\left[t_{0}, t_{1}\right)$. A solution $x(t)$ of (1) is called oscillatory if there exists a sequence $\left\{\lambda_{n}\right\}_{n=1}^{\infty}$ of points in the interval $\left[t_{0}, \infty\right)$, so that $\lim _{n \rightarrow \infty} \lambda_{n}=\infty$ and $x\left(\lambda_{n}\right)=0, n \in \mathbb{N}$; otherwise it is called nonoscillatory. Equation (1) is called oscillatory if all its solutions are oscillatory.

We note that if $f\left(x^{\prime}(t)\right)=x^{\prime}(t)$ and $H\left(t, x(t), x^{\prime}(t)\right)=0$, then (1) becomes the differential equation of the form

$$
\begin{array}{r}
\left(r(t) \psi(x(t)) x^{\prime}(t)\right)^{\prime}+q(t) g(x(t))=0, \\
t \in\left[t_{0}, \infty\right), \quad t_{0}>0 .
\end{array}
$$

The oscillation of solutions of (2) has been studied by Elsheikh [1], Manojlovic [10], and Ohriska and Zulova [5] under different conditions. Also, if $\psi(x(t))=1, f\left(x^{\prime}(t)\right)=x^{\prime}(t)$ and $q(t) g(x(t))=Q(t, x)$, then (1) becomes the differential equation of the form

$$
\begin{array}{r}
\left(r(t) x^{\prime}(t)\right)^{\prime}+Q(t, x)=H\left(t, x^{\prime}(t), x(t)\right), \\
t \in\left[t_{0}, \infty\right), \quad t_{0}>0 .
\end{array}
$$

The oscillation of solutions of (3) has been studied by Remili $[7,8]$, where new results with additional suitable weighted function are found. Recently, Temtek and Tiryaki 
[9] obtained several new oscillation criteria for (3) with $x^{\prime}(t)=\left|x^{\prime}(t)\right|^{\alpha-1} x^{\prime}(t)$ by using generalized Riccati transformation and well-known techniques, such as averaging technique.

The purpose of this research is to remove the above mentioned conditions which depend on the solution and extend some results presented in $[1,5,7,8]$.

Although Grace [2] considered the function $H$ defined by $H(t, s)=\left(\int_{t_{0}}^{t}(d s / \Theta(s))\right)^{\alpha}, t \geq t_{0}>0$ for some constant $\alpha>1$ and there is a positive continuous function $\Theta$ on $\left[t_{0}, \infty\right)$ such that $\int_{t_{0}}^{\infty}(d s / \Theta(s))=\infty$, Kirane and Rogovchenko [11] and Tiryaki and Ayanlar [12] also mentioned that they can obtain new oscillation criteria with the a particular choice of the functions $H$ and $h$ with $H(t, s)=\left(\int_{t_{0}}^{t}(d s / \Theta(s))\right)^{\alpha-1}, t \geq t_{0}>0$ and $\alpha>2, \int_{t_{0}}^{\infty}(d s / \Theta(s))=\infty$, and Li [13] defined $H(t, s)=$ $(R(t)-R(s))^{\alpha}, \alpha>1, R(t)=\int_{t_{0}}^{\infty}(d s / r(s)), t \geq t_{0}$. However, according to Ohriska and Zulova [5], research which involves oscillatory criteria based on the weight function $H(t, s)$ has increased. These results have great theoretical value but they are less effective in applications. On the other hand, the results which contain the requirements only on the functions occurring in differential equation are usually better applicable. In particular, our weight function $H(t, s)$ is not strictly Philos-type function because in the present paper, we ignored Philos's conditions (see $[2,6,10,11,14-17]$. This paper contains only results of the latter kind by restricting only the functions in the differential equation (1). The relevance of the theorems in this paper is illustrated by examples.

\section{Main Results}

In this section we give some new sufficient conditions for all solutions of (1) to be oscillatory. We introduce the following notation:

$$
\begin{gathered}
R(t)=\int_{t_{0}}^{t} \frac{d s}{r(s)}, \quad t \geq t_{0}, \\
Z(t)=\rho(t)[q(t)-p(t)]-\frac{1}{4 A} \frac{\left(\rho^{\prime}(t)\right)^{2}}{\rho(t)} r(t) .
\end{gathered}
$$

By Theorems 1 and 3, the given conditions include Kamenev's integral criteria.

Theorem 1. One assumes that there are positive constants $k$ and $l$ satisfying

$$
\begin{gathered}
\frac{g^{\prime}(x)}{\psi(x)} \geq k>0, \quad \forall x \neq 0, \\
f^{2}(y) \leq \operatorname{lyf}(y), \quad \forall y \in \mathbb{R} .
\end{gathered}
$$

Furthermore let, for some integer $m \geq 3$,

$$
\begin{aligned}
\lim _{t \rightarrow \infty} & \sup \frac{1}{R^{m-1}(t)} \int_{t_{0}}^{t}[R(t)-R(s)]^{m-1}(q(s)-p(s)) d s \\
& =\infty .
\end{aligned}
$$

\section{Then (1) is oscillatory.}

Proof. Assume, for the sake of a contradiction, that (1) has an eventually positive solution $x(t)>0$, for all $T \geq t_{0}>0$. The case $x(t)$ being eventually negative can be similarly discussed. Set

$$
w(t)=\frac{r(t) \psi(x(t)) f\left(x^{\prime}(t)\right)}{g(x(t))}, \quad t \geq T .
$$

Then by $\left(A_{4}\right),(1)$, and (5), we have

$$
\begin{aligned}
& w^{\prime}(t)= \frac{\left[r(t) \psi(x(t)) f\left(x^{\prime}(t)\right)\right]^{\prime}}{g(x(t))} \\
&-\frac{r(t) \psi(x(t)) f\left(x^{\prime}(t)\right) x^{\prime}(t) g^{\prime}(x(t))}{g^{2}(x(t))}, \\
& w^{\prime}(t)+q(t)-p(t)+\frac{k}{l} \frac{w^{2}(t)}{r(t)} \leq 0, \quad t \geq T .
\end{aligned}
$$

Hence, for all $t \geq s \geq T$, it can be written as

$$
\begin{gathered}
\int_{T}^{t}(R(t)-R(s))^{m-1}[q(s)-p(s)] d s \\
\leq-\int_{T}^{t}(R(t)-R(s))^{m-1} w^{\prime}(s) d s \\
-\frac{k}{l} \int_{T}^{t}(R(t)-R(s))^{m-1} \frac{w^{2}(s)}{r(s)} d s \\
=w(T)(R(t)-R(T))^{m-1} \\
\quad-\int_{T}^{t}\left[\frac{(m-1)}{r(s)}(R(t)-R(s))^{m-2} w(s)\right. \\
\left.\quad+\frac{k(R(t)-R(s))^{m-1}}{\operatorname{lr}(s)} w^{2}(s)\right] d s .
\end{gathered}
$$


Then (9) implies that

$$
\begin{aligned}
\int_{T}^{t}(R(t) & -R(s))^{m-1}[q(s)-p(s)] d s \\
\leq & w(T)(R(t)-R(T))^{m-1}-\frac{l(m-1)^{2}}{4 k} \\
\quad & \int_{T}^{t}(R(t)-R(s))^{m-3} \frac{1}{r(s)} d s \\
- & \int_{T}^{t} \frac{(R(t)-R(s))^{m-3}}{r(s)} \\
& \times\left\{\sqrt{\frac{k}{l}}(R(t)-R(s)) w(s)+\frac{m-1}{2 \sqrt{k / l}}\right\}^{2} d s .
\end{aligned}
$$

Then we have

$$
\begin{aligned}
\frac{1}{R^{m-1}(t)} \int_{T}^{t}(R(t)-R(s))^{m-1}[q(s)-p(s)] d s & \\
\leq & {\left[1-\frac{R(T)}{R(t)}\right]^{m-1} w(T) } \\
& +\frac{l(m-1)^{2}}{4 k(m-2) R(t)}\left[1-\frac{R(T)}{R(t)}\right]^{m-2}, \quad t \geq T .
\end{aligned}
$$

It is clear that, for $t \in\left[t_{0}, T\right]$, it holds

$$
\begin{aligned}
& \frac{1}{R^{m-1}(t)} \int_{t_{0}}^{T}(R(t)-R(s))^{m-1}[q(s)-p(s)] d s \\
& \quad \leq \frac{1}{R^{m-1}(t)} \int_{t_{0}}^{T} R^{m-1}(t)[q(s)-p(s)] d s \\
& \quad=\int_{t_{0}}^{T}[q(s)-p(s)] d s .
\end{aligned}
$$

Combining (11) and (12), it follows that

$$
\begin{gathered}
\frac{1}{R^{m-1}(t)} \int_{t_{0}}^{t}(R(t)-R(s))^{m-1}[q(s)-p(s)] d s \\
\leq\left[1-\frac{R(T)}{R(t)}\right]^{m-1} w(T) \\
\quad+\frac{l(m-1)^{2}}{4 k(m-2) R(t)}\left[1-\frac{R(T)}{R(t)}\right]^{m-2} \\
\quad+\int_{t_{0}}^{T}[q(s)-p(s)] d s, \quad t \geq t_{0} .
\end{gathered}
$$

It is observable that $\lim _{t \rightarrow \infty}(1 / R(t))=N \in[0, \infty)$. Thus

$$
\begin{aligned}
\lim _{t \rightarrow \infty} \sup & \frac{1}{R^{m-1}(t)} \int_{t_{0}}^{t}(R(t)-R(s))^{m-1}[q(s)-p(s)] d s \\
\leq & {[1-N R(T)]^{m-1} w(T) } \\
& +\frac{N l(m-1)^{2}}{4 k(m-2) R(t)}[1-N R(T)]^{m-2} \\
& +\int_{t_{0}}^{T}[q(s)-p(s)] d s<\infty,
\end{aligned}
$$

which contradicts condition (6). The proof of the theorem is completed.

Example 2. Consider the differential equation

$$
\begin{gathered}
{\left[\frac{1}{t}\left(\frac{1}{x^{2}(t)+1}\right) x^{\prime}(t)\right]^{\prime}+3 x(t)\left(2+\frac{1}{1+x^{2}(t)}\right)} \\
=\frac{x(t)}{4}\left(2+\frac{1}{1+x^{2}(t)}\right) \sin \frac{\left(x^{\prime}(t)\right)^{2}}{\left(x^{\prime}(t)\right)^{2}+1} .
\end{gathered}
$$

Clearly,

$$
\begin{gathered}
\frac{g^{\prime}(x)}{\psi(x)}=3+\frac{2 x^{4}}{1+x^{2}} \geq 3=k, \\
f^{2}(y)=y^{2}+\frac{2 y^{2}}{1+y^{2}}+\frac{y^{2}}{\left(1+y^{2}\right)^{2}} \leq 4 y^{2}, \\
\frac{1}{\frac{1}{4} f^{2}(y) \leq y^{2} \leq} y^{2}+\frac{y^{2}}{y^{2}+1}=y f(y) \quad \forall y \in \mathbb{R}, \\
\frac{g\left(t, x^{\prime}(t), x(t)\right)}{\left.g^{2}(t)\right)}=\frac{x(t)}{4}\left(2+\frac{1}{1+x^{2}(t)}\right) \sin \frac{\left(x^{\prime}(t)\right)^{2}}{\left(x^{\prime}(t)\right)^{2}+1} \\
\times \frac{1}{x(t)\left(1+x^{2}(t)\right)^{2}} \\
\leq \frac{1}{4}=p(t),
\end{gathered}
$$

and, for any integer $m \geq 3$, we have

$$
\lim _{t \rightarrow \infty} \sup \frac{1}{R^{m-1}(t)} \int_{t_{0}}^{t}[R(t)-R(s)]^{m-1}(q(s)-p(s)) d s
$$




$$
=4 \lim _{t \rightarrow \infty} \sup \frac{1}{t^{4}} \int_{t_{0}}^{t}\left[\frac{t^{2}}{2}-\frac{s^{2}}{2}\right]^{2}\left(3-\frac{1}{4}\right) d s=\infty .
$$

By Theorem 1, this equation is oscillatory.

Theorem 3. Assume that (5) holds and suppose that

$$
\begin{gathered}
\lim _{t \rightarrow \infty} \sup \frac{1}{t^{m}} \int_{t_{0}}^{t}(t-s)^{m}(q(s)-p(s)) \\
-\frac{l^{2}}{4 k}(t-s)^{m-2} r(s) d s=\infty,
\end{gathered}
$$

for some $m \geq 2$. Then (1) is oscillatory.

Proof. Assume that (1) has an eventually positive solution $x(t)>0$, for all $T \geq t_{0}>0$. Taking $w(t)$ as it is defined in (7), we obtain (8).

Hence by $\left(\mathrm{A}_{3}\right)$, for $t \geq s \geq T$, we can write

$$
\begin{aligned}
\int_{T}^{t}(t & -s)^{m}[q(s)-p(s)] d s \\
& \leq-\int_{T}^{t}(t-s)^{m} w^{\prime}(s) d s-\frac{k}{l} \int_{T}^{t}(t-s)^{m} \frac{w^{2}(s)}{r(s)} d s .
\end{aligned}
$$

That is,

$$
\begin{aligned}
& \int_{T}^{t}(t-s)^{m}[q(s)-p(s)] d s \\
& \leq(t-T)^{m} w(T)-m \int_{T}^{t}(t-s)^{m-1} w(s) d s \\
& -\frac{k}{l} \int_{T}^{t}(t-s)^{m} \frac{w^{2}(s)}{r(s)} d s=(t-T)^{m} w(T) \\
& -\int_{T}^{t}\left[\sqrt{\frac{k(t-s)^{m}}{l r(s)}} w(s)+\frac{m}{2} \sqrt{\frac{\operatorname{lr}(s)(t-s)^{m-2}}{k}}\right]^{2} d s \\
& +\frac{\operatorname{lm}^{2}}{4 k} \int_{T}^{t} r(s)(t-s)^{m-2} d s .
\end{aligned}
$$

Hence it follows that

$$
\begin{gathered}
\int_{T}^{t}\left((t-s)^{m}[q(s)-p(s)]-\frac{\operatorname{lm}^{2}}{4 k} r(s)(t-s)^{m-2}\right) d s \\
\leq(t-T)^{m} w(T) \leq\left(t-t_{0}\right)^{m} w(T), \quad t \geq T .
\end{gathered}
$$

Using the inequality (21), for $t \geq t_{0}$, we obtained

$$
\begin{gathered}
\int_{T}^{t}\left((t-s)^{m}[q(s)-p(s)]-\frac{l m^{2}}{4 k} r(s)(t-s)^{m-2}\right) d s \\
=\int_{t_{0}}^{T}\left((t-s)^{m}[q(s)-p(s)]-\frac{l^{2}}{4 k} r(s)(t-s)^{m-2}\right) d s \\
\quad+\int_{T}^{t}\left((t-s)^{m}[q(s)-p(s)]-\frac{l^{2}}{4 k} r(s)(t-s)^{m-2}\right) d s, \\
\int_{t_{0}}^{t}\left((t-s)^{m}[q(s)-p(s)]-\frac{l m^{2}}{4 k} r(s)(t-s)^{m-2}\right) d s \\
\leq\left(t-t_{0}\right)^{m} w(T) \int_{t_{0}}^{t}(q(s)-p(s)) d s+\left(t-t_{0}\right)^{m} w(T) .
\end{gathered}
$$

Thus,

$$
\begin{array}{r}
\lim _{t \rightarrow \infty} \sup \frac{1}{t^{m}} \int_{t_{0}}^{t}\left((t-s)^{m}[q(s)-p(s)]\right. \\
\left.\quad-\frac{\operatorname{lm}^{2}}{4 k} r(s)(t-s)^{m-2}\right) d s \\
\leq \int_{t_{0}}^{t}(q(s)-p(s)) d s+w(T)<\infty,
\end{array}
$$

and it contradicts (18).

Example 4. Consider the differential equation

$$
\begin{gathered}
{\left[\left(\frac{1+(x(t))^{2}}{2+(x(t))^{2}}\right) x^{\prime}(t)\left(\frac{1+2\left(x^{\prime}(t)\right)^{2}}{4+2\left(x^{\prime}(t)\right)^{2}}\right)\right]^{\prime}+(1+\cos t) x(t)} \\
=\frac{x^{5}(t)}{x^{2}(t)+1} \cos t \frac{\left(x^{\prime}(t)\right)^{2}}{\left(x^{\prime}(t)\right)^{2}+1}, \quad t>0,
\end{gathered}
$$

which satisfies the conditions of Theorem 3, since we have

$$
\begin{gathered}
\frac{g^{\prime}(x)}{\psi(x)}=\frac{2+(x(t))^{2}}{1+(x(t))^{2}} \geq 2=k \quad \forall x \neq 0, \\
y f(y)=y^{2} \frac{1+2 y^{2}}{4+2 y^{2}}>0, \quad f^{2}(y) \leq L y f(y), \quad L=1, \\
\frac{H\left(t, x^{\prime}(t), x(t)\right)}{g(x(t))}=\frac{x^{5}(t)}{x^{2}(t)+1} \cos t \frac{\left(x^{\prime}(t)\right)^{2}}{\left(x^{\prime}(t)\right)^{2}+1} \times \frac{1}{x^{3}(t)} \\
\leq \cos t=p(t), \quad \forall x^{\prime} \in \mathbb{R}, \quad x \in \mathbb{R}, t \geq t_{0} ;
\end{gathered}
$$


and, for any integer $m \geq 2$, we have

$$
\begin{gathered}
\lim _{t \rightarrow \infty} \sup \frac{1}{t^{m}} \int_{t_{0}}^{t}\left((t-s)^{m}[1+\cos (s)-\cos (s)]\right. \\
\left.\quad-\frac{m^{2}}{8}(t-s)^{m-2}\right) d s \\
=\lim _{t \rightarrow \infty} \sup \frac{1}{t^{m}}\left[\frac{t}{(m+1)}-\frac{m^{2}}{8(m-1) t}\right]=\infty .
\end{gathered}
$$

It thus follows that every solution of the given equation oscillates.

\section{Theorem 5. Suppose that}

$$
\begin{gathered}
0<k_{2} \leq \psi(x(t)) \leq k_{3}, \quad \forall x \in \mathbb{R}, \\
0<k_{4} \leq \frac{f(y)}{y} \leq k_{5}, \quad \forall y=x^{\prime}(t) \neq 0 .
\end{gathered}
$$

Let $\rho$ be a positive continuously differentiable function over $[T, \infty)$ such that $\rho^{\prime}(t) \geq 0$ over $[T, \infty)$,

$$
\begin{gathered}
\lim _{t \rightarrow \infty} \int_{T_{0}}^{t} \frac{1}{\rho(s) r(s)} d s=\infty, \\
\lim _{t \rightarrow \infty} \int_{T_{0}}^{t} Z(s) d s=\infty .
\end{gathered}
$$

Then all solutions of (1) are oscillatory.

Proof. Let $x(t)$ be a nonoscillatory solution on $[T, \infty), T \geq T_{0}$ of (1). We assume that $x(t)$ is positive on $[T, \infty)$. A similar argument holds for the case when $x(t)$ is negative. Let

$$
w(t)=\frac{r(t) \psi(x(t)) f\left(x^{\prime}(t)\right)}{g(x(t))} .
$$

Then

$$
w^{\prime}(t)=\left[\frac{r(t) \psi(x(t)) f\left(x^{\prime}(t)\right)}{g(x(t))}\right]^{\prime} .
$$

Using (1), we obtain

$$
\begin{aligned}
w^{\prime}(t)= & \frac{H\left(t, x^{\prime}(t), x(t)\right)}{g(x(t))} \\
& -q(t)-\frac{r(t) \psi(x(t)) f\left(x^{\prime}(t)\right) g^{\prime}(x(t)) x^{\prime}(t)}{g^{2}(x(t))} .
\end{aligned}
$$

Since $g^{\prime}(x(t))>k$ and condition $\left(\mathrm{A}_{4}\right)$ we obtained,

$$
\begin{aligned}
w^{\prime}(t) \leq & p(t)-q(t) \\
& -\frac{k}{r(t) \psi(x(t))} \frac{r^{2}(t) \psi^{2}(x(t))\left(f\left(x^{\prime}(t)\right)\right)^{2}}{g^{2}(x(t))} \\
& \times \frac{x^{\prime}(t)}{f\left(x^{\prime}(t)\right)} .
\end{aligned}
$$

From (27) and (30),

$$
w^{\prime}(t) \leq-[q(t)-p(t)]-\frac{k}{k_{3} k_{4}} \frac{1}{r(t)} w^{2}(t) .
$$

Therefore,

$$
\begin{aligned}
{\left[\frac{r(t) \psi(x(t)) f\left(x^{\prime}(t)\right)}{g(x(t))}\right]^{\prime} \leq } & -[q(t)-p(t)] \\
& -\frac{k}{k_{3} k_{4}} \frac{1}{r(t)} w^{2}(t) .
\end{aligned}
$$

Multiplying by $\rho(t)$ and integrating from $T$ to $t$, we obtained

$$
\begin{gathered}
\rho(t)\left[\frac{r(t) \psi(x(t)) f\left(x^{\prime}(t)\right)}{g(x(t))}\right]^{\prime} \\
\leq-\rho(t)[q(t)-p(t)] \\
-A \frac{\rho(t)}{r(t)} w^{2}(t) ; \quad A=\frac{k}{k_{3} k_{4}}, \\
\int_{T}^{t} \rho(s)\left[\frac{r(s) \psi(x(s)) f\left(x^{\prime}(s)\right)}{g(x(s))}\right]^{\prime} d s \\
\leq \int_{T}^{t}-\rho(s)[q(s)-p(s)] d s \\
-\int_{T}^{t} A \frac{\rho(s)}{r(s)} w^{2}(s) d s .
\end{gathered}
$$

Let

$$
C_{T}=\frac{\rho(T) r(T) \psi(x(T)) f\left(x^{\prime}(T)\right)}{g(x(T))} .
$$

By integrating by parts, we obtained

$$
\begin{aligned}
\frac{\rho(t) r(t) \psi(x(t)) f\left(x^{\prime}(t)\right)}{g(x(t))} \\
\leq C_{T}-\int_{T}^{t} \rho(s)[q(s)-p(s)] d s \\
\quad-\int_{T}^{t} A \frac{\rho(s)}{r(s)} w^{2}(s) d s \\
\quad+\int_{T}^{t} \rho^{\prime}(s) \frac{r(s) \psi(x(s)) f\left(x^{\prime}(s)\right)}{g(x(s))} d s \\
=C_{T}-\int_{T}^{t} \rho(s)[q(s)-p(s)] d s \\
\quad-\int_{T}^{t} A \frac{\rho(s)}{r(s)} w^{2}(s) d s+\int_{T}^{t} \rho^{\prime}(s) w(s) d s \\
=C_{T}-\int_{T}^{t} \rho(s)[q(s)-p(s)] d s \\
+\int_{T}^{t}\left[-A^{\rho} \frac{\rho(s)}{r(s)} w^{2}(s)+\rho^{\prime}(s) w(s)\right] d s .
\end{aligned}
$$


The inequality presented by (38) can be written as

$$
\begin{aligned}
& \frac{\rho(t) r(t) \psi(x(t)) f\left(x^{\prime}(t)\right)}{g(x(t))} \\
& \leq C_{T}-\int_{T}^{t} \rho(s)[q(s)-p(s)] d s \\
& \quad+\int_{T}^{t} \frac{-A \rho(s)}{r(s)} \\
& \quad \times\left[\left(w(s)-\frac{\rho^{\prime}(s) r(s)}{2 A \rho(s)}\right)^{2}-\frac{\left(\rho^{\prime}(s)\right)^{2} r^{2}(s)}{4 A^{2} \rho^{2}(s)}\right] d s .
\end{aligned}
$$

Let $w(s)-\left(\left(\rho^{\prime}(s) r(s)\right) /(2 A \rho(s))\right)=W(s)$,

$$
\begin{aligned}
& \frac{\rho(t) r(t) \psi(x(t)) f\left(x^{\prime}(t)\right)}{g(x(t))} \\
& \leq C_{T}-\int_{T}^{t} \rho(s)[q(s)-p(s)] d s \\
&-\int_{T}^{t} \frac{A \rho(s)}{r(s)}\left[W^{2}(s)-\left(\frac{\rho^{\prime}(s) r(s)}{2 A \rho(s)}\right)^{2}\right] d s \\
&= C_{T}-\int_{T}^{t} \rho(s)[q(s)-p(s)] d s \\
&-\int_{T}^{t}\left[\frac{A \rho(s)}{r(s)} W^{2}(s)-\frac{\rho^{\prime 2}(s) r(s)}{4 A \rho(s)}\right] d s \\
&= C_{T}-\int_{T}^{t} \rho(s)[q(s)-p(s)]-\frac{1}{4 A} \frac{\left(\rho^{\prime}(s)\right)^{2}}{\rho(s)} r(s) d s \\
&-\int_{T}^{t} \frac{A \rho(s)}{r(s)} W^{2}(s) d s, \\
& \frac{\rho(t) r(t) \psi(x(t)) f\left(x^{\prime}(t)\right)}{g(x(t))} \leq C_{T}-\int_{T}^{t} Z(s) d s .
\end{aligned}
$$

Taking the limit for both sides of (40) and using (29),

$$
\lim _{t \rightarrow \infty} \frac{\rho(t) r(t) \psi(x(t)) f\left(x^{\prime}(t)\right)}{g(x(t))} \longrightarrow-\infty .
$$

Hence, there exists $T_{1} \geq T$ such that

$$
\begin{gathered}
f\left(x^{\prime}(t)\right)<0, \quad \forall t \geq T_{1}, \\
x^{\prime}(t)<0, \quad \forall t \geq T_{1} .
\end{gathered}
$$

Condition (29) also implies that $\int_{T}^{\infty} \rho(s)[q(s)-p(s)] d s=\infty$, and there exists $T_{2} \geq T_{1}$ such that

$$
\begin{gathered}
\int_{T_{1}}^{T_{2}} \rho(s)[q(s)-p(s)] d s=0, \\
\int_{T_{2}}^{t} \rho(s)[q(s)-p(s)] d s \geq 0, \quad \forall t \geq T_{2} .
\end{gathered}
$$

Multiplying (1) by $\rho(t)$ and integrating by parts on $\left[T_{2}, t\right]$, we obtained

$$
\begin{aligned}
\rho(t) & {\left[r(t) \psi(x(t)) f\left(x^{\prime}(t)\right)\right]^{\prime}+\rho(t) q(t) g(x(t)) } \\
& =\rho(t) H\left(t, x^{\prime}(t), x(t)\right),
\end{aligned}
$$

which implies

$$
\begin{gathered}
\frac{\rho(t)\left[r(t) \psi(x(t)) f\left(x^{\prime}(t)\right)\right]^{\prime}}{g(x(t))} \leq-\rho(t)[q(t)-p(t)] \\
\rho(t)\left[r(t) \psi(x(t)) f\left(x^{\prime}(t)\right)\right]^{\prime} \\
\leq-\rho(t) g(x(t))[q(t)-p(t)] .
\end{gathered}
$$

Now, integrating by parts, we get

$$
\begin{aligned}
& \rho(t)\left[r(t) \psi(x(t)) f\left(x^{\prime}(t)\right)\right] \\
& \quad-\int_{T_{2}}^{t} \rho^{\prime}(s) r(s) \psi(x(s)) f\left(x^{\prime}(s)\right) d s \\
& \leq C_{T_{2}}-\int_{T_{2}}^{t} \rho(s) g(x(s))[q(s)-p(s)] d s \\
&=C_{T_{2}}-g(x(t)) \int_{T_{2}}^{t} \rho(s)[q(s)-p(s)] d s \\
& \quad+\int_{T_{2}}^{t} x^{\prime}(s) g^{\prime}(x(s)) \int_{T_{2}}^{s} \rho(u)[q(u)-p(u)] d u d s \\
& \quad+\int_{T_{2}}^{t} \rho^{\prime}(s) r(s) \psi(x(s)) f\left(x^{\prime}(s)\right) d s \\
& \leq C_{T_{2}}, \quad \forall t \geq T_{1},
\end{aligned}
$$

where $C_{T_{2}}=\left(\rho\left(T_{2}\right) r\left(T_{2}\right) \psi\left(x\left(T_{2}\right)\right) f\left(x^{\prime}\left(T_{2}\right)\right)\right) /\left(g\left(x\left(T_{2}\right)\right)\right)<0$.

Therefore,

$$
\rho(t) r(t) \psi(x(t)) f\left(x^{\prime}(t)\right) \leq C_{T_{2}} .
$$

From (27), we have

$$
x^{\prime}(t) \leq \frac{C_{T_{2}}}{k_{2} k_{4}} \frac{1}{r(t) \rho(t)}, \quad x(t) \leq \frac{C_{T_{2}}}{k_{2} k_{4}} \int_{T_{2}}^{t} \frac{1}{r(s) \rho(s)} d s .
$$

Finally, from (28), $x(t) \rightarrow-\infty$ as $t \rightarrow \infty$, which is a contradiction. 
Example 6. Let us consider the following equation:

$$
\begin{aligned}
& {\left[\frac{1}{t}\left(5+\frac{x^{6}(t)}{x^{6}(t)+1}\right)\left(13 x^{\prime}(t)+\frac{x^{\prime}(t)}{\left(x^{\prime}(t)\right)^{2}+1}\right)\right]^{\prime}} \\
& +\left(t+\frac{\sin t}{t}\right) x^{5}(t)=\frac{2 x^{12} \sin t \cos \left(x^{\prime}(t)+1\right)}{\left(x^{7}+1\right) t^{3}}, \\
& t \geq \frac{\pi}{2} .
\end{aligned}
$$

We note that

$$
\begin{aligned}
& \frac{H\left(t, x^{\prime}(t), x(t)\right)}{g(x(t))} \\
& =\frac{2 x^{12} \sin t \cos \left(x^{\prime}(t)+1\right)}{\left(x^{7}+1\right) t^{3}} \times \frac{1}{x^{5}(t)} \leq \frac{2}{t^{3}}=p(t), \\
& \forall x^{\prime} \in \mathbb{R}, \quad x \in \mathbb{R}, \quad t \geq t_{0} .
\end{aligned}
$$

Hence,

$$
\begin{gathered}
r(t)=\frac{1}{t}>0, \quad t>\frac{\pi}{2}, \\
5 \leq 5+\frac{x^{6}(t)}{x^{6}(t)+1}<6, \quad \forall x \in \mathbb{R}, \\
13<13+\frac{1}{\left(x^{\prime}(t)\right)^{2}+1} \leq 14, \quad \forall y \neq 0 .
\end{gathered}
$$

Let

$$
\begin{gathered}
\rho(t)=t \Longrightarrow \rho^{\prime}(t)=1 \\
\int_{t}^{\infty} \frac{d s}{r(s) \rho(s)}=\int_{t}^{\infty} d s=\infty \\
Z(s)=\rho(s)[q(s)-p(s)]-\frac{1}{4 A} \frac{\left(\rho^{\prime}(s)\right)^{2}}{\rho(s)} r(s) \\
=s\left[s+\frac{\sin s}{s}-\frac{2}{s^{3}}\right]-\frac{1}{4 A} \frac{1}{s^{2}}
\end{gathered}
$$

For every $t \geq T=\pi / 2$, we obtained

$$
\int_{t_{0}}^{\infty} Z(s) d s=\int_{t_{0}}^{\infty} s^{2}+\sin s-\frac{2}{s^{2}}-\frac{1}{4 A s^{2}} d s=\infty .
$$

Then, Theorem 5 ensures that every solution in this example is oscillatory.

Theorem 7. If conditions (27)-(29) hold and

$$
\begin{gathered}
\int_{T_{0}}^{\infty} \rho(s)[q(s)-p(s)] d s<\infty, \\
\lim _{t \rightarrow \infty} \inf \left[\int_{T}^{t} Z(s) d s\right] \geq 0, \quad \forall \text { large } T, \\
\lim _{t \rightarrow \infty} \int_{T_{0}}^{t} \frac{1}{\rho(s) r(s)} \int_{s}^{\infty} Z(u) d u d s=\infty,
\end{gathered}
$$

$$
\int_{\varepsilon}^{\infty} \frac{d y}{g(y)}<\infty, \quad \int_{-\varepsilon}^{-\infty} \frac{d y}{g(y)}<\infty \quad \text { for every } \varepsilon>0,
$$

thus all solutions of (1) are oscillatory.

Remark 8. Condition (55) implies that $\int_{T}^{\infty} Z(s) \geq 0$ and $\liminf _{t \rightarrow \infty} \int_{T}^{\infty} Z(s) d s=\int_{T}^{\infty} Z(s) d s$; hence, (56) takes the form of $\int_{T}^{\infty} Z(s) \geq 0$, for all large $T$.

Proof. Let $x(t)$ be a nonoscillatory solution on $[T, \infty), T \geq$ $T_{0}$ of (1). Let us assume that $x(t)$ is positive on $[T, \infty)$ and consider the following three cases for the behavior of $x^{\prime}(t)$.

Case 1. Consider $x^{\prime}(t)>0$ for $T_{1} \geq T$ for some $t \geq T_{1}$; then, from (40), we have

$$
\begin{aligned}
\int_{T_{1}}^{t} R(s) d s \leq & \frac{r\left(T_{1}\right) \rho\left(T_{1}\right) \psi\left(x\left(T_{1}\right)\right) f\left(x^{\prime}\left(T_{1}\right)\right)}{g\left(x\left(T_{1}\right)\right)} \\
& -\frac{\rho(t) r(t) \psi(x(t)) f\left(x^{\prime}(t)\right)}{g(x(t))}, \\
k_{4} k_{2} \frac{r(t) \rho(t) x^{\prime}(t)}{g(x(t))} \leq & \frac{r\left(T_{1}\right) \rho\left(T_{1}\right) \psi\left(x\left(T_{1}\right)\right) f\left(x^{\prime}(t)\right)}{g\left(x\left(T_{1}\right)\right)} \\
& -\int_{T_{1}}^{t} R(s) d s .
\end{aligned}
$$

From (27), we obtained

$$
\begin{aligned}
\int_{T_{1}}^{t} Z(s) d s \leq & \frac{r\left(T_{1}\right) \rho\left(T_{1}\right) \psi\left(x\left(T_{1}\right)\right) f\left(x^{\prime}\left(T_{1}\right)\right)}{g\left(x\left(T_{1}\right)\right)} \\
& -k_{4} k_{2} \frac{r(t) \rho(t) x^{\prime}(t)}{g(x(t))} .
\end{aligned}
$$

Hence, for all $t \geq T_{1}$,

$$
\begin{gathered}
\int_{t}^{\infty} R(s) d s \leq k_{4} k_{2} \frac{r(t) \rho(t) x^{\prime}(t)}{g(x(t))}, \\
\int_{T_{1}}^{t} \frac{1}{r(s) \rho(s)} \int_{s}^{\infty} Z(u) d u d s \leq k_{4} k_{2} \int_{T_{1}}^{\infty} \frac{x^{\prime}(s)}{g(x(s))} d s, \\
\int_{T_{1}}^{t} \frac{1}{r(s) \rho(s)} \int_{s}^{\infty} Z(u) d u d s \leq k_{4} k_{2} \int_{T_{1}(s)}^{\infty} \frac{d y}{g(y)} .
\end{gathered}
$$

Using (57), we obtained

$$
\int_{T_{1}}^{t} \frac{1}{r(s) \rho(s)} \int_{s}^{\infty} Z(u) d u d s<\infty .
$$

This contradicts condition (56).

Case 2. If $x^{\prime}(t)$ is oscillatory, then there exists a sequence $\left\{\alpha_{n}\right\} \rightarrow \infty$ on $[T, \infty)$ such that $x^{\prime}\left(\alpha_{n}\right)<0$. Let us assume that $N$ is sufficiently large, so that

$$
\int_{\alpha_{N}}^{\infty} Z(s) d s \geq 0
$$


Then, from (27) and (40), we have

$$
k_{3} k_{5} \frac{\rho(t) r(t) x^{\prime}(t)}{g(x(t))} \leq C_{\alpha_{N}}-\int_{\alpha_{N}}^{t} Z(s) d s
$$

Thus,

$$
\begin{aligned}
k_{3} k_{5} \limsup _{t \rightarrow \infty} \frac{\rho(t) r(t) x^{\prime}(t)}{g(x(t))} & \leq C_{\alpha_{N}}+\limsup _{t \rightarrow \infty}\left[-\int_{\alpha_{N}}^{t} Z(s) d s\right] \\
& =C_{\alpha_{N}}-\liminf _{t \rightarrow \infty}\left[\int_{\alpha_{N}}^{t} Z(s) d s\right] .
\end{aligned}
$$

By (55), we obtained

$$
k_{3} k_{5} \limsup _{t \rightarrow \infty} \frac{\rho(t) r(t) x^{\prime}(t)}{g(x(t))}<0
$$

which contradicts the fact that $x^{\prime}(t)$ oscillates.

Case 3. Let $x^{\prime}(t)<0$ for $t \geq T$ for some $T_{1} \geq T$; then, for any $t_{0} \geq T_{0}$, there exists $t_{1} \geq t_{0}$ such that $\int_{t_{1}}^{\infty} \rho(s)[q(s)-p(s)] d s \geq$ 0 for all $t \geq t_{1}$. Choosing $t_{1} \geq T_{1}$, and multiplying (1) by $\rho(t)$ and integrating by parts, we obtained

$$
\begin{aligned}
& \rho(t)\left[r(t) \psi(x(t)) f\left(x^{\prime}(t)\right)\right] \\
& \quad-\int_{t_{1}}^{t} \rho^{\prime}(s) r(s) \psi(x(s)) f\left(x^{\prime}(s)\right) d s \\
& \leq C_{t_{1}}-\int_{t_{1}}^{t} \rho(s) g(x(s))[q(s)-p(s)] d s \\
& =C_{t_{1}}-g(x(t)) \int_{t_{1}}^{t} \rho(s)[q(s)-p(s)] d s \\
& \quad+\int_{t_{1}}^{t} x^{\prime}(s) g^{\prime}(x(s)) \int_{t_{1}}^{s} \rho(u)[q(u)-p(u)] d u d s \\
& \quad+\int_{t_{1}}^{t} \rho^{\prime}(s) r(s) \psi(x(s)) f\left(x^{\prime}(s)\right) d s,
\end{aligned}
$$

where $C_{t_{1}}=\rho\left(t_{1}\right) r\left(t_{1}\right) \psi\left(x\left(t_{1}\right)\right) f\left(x^{\prime}\left(t_{1}\right)\right)<0$.

Thus,

$$
\rho(t) r(t) \psi(x(t)) f\left(x^{\prime}(t)\right) \leq C_{t_{1}} .
$$

From (27), we obtained

$$
\begin{gathered}
x^{\prime}(t) \leq \frac{C_{t_{1}}}{k_{2} k_{4}} \frac{1}{r(t) \rho(t)}, \\
x(t) \leq \frac{C_{t_{1}}}{k_{2} k_{4}} \int_{T_{2}}^{t} \frac{1}{r(s) \rho(s)} d s .
\end{gathered}
$$

From (28), it follows that $x(t) \rightarrow-\infty$, as $t \rightarrow \infty$, which is a contradiction.
Example 9. Consider the following equation:

$$
\begin{gathered}
{\left[t\left(8+\frac{x^{4}(t)}{x^{4}(t)+1}\right)\left(7 x^{\prime}(t)+\frac{\left(x^{\prime}(t)\right)^{5}}{\left(x^{\prime}(t)\right)^{4}+1}\right)\right]^{\prime}} \\
+\frac{1}{t^{3}} x^{3}(t)=\frac{x^{3} \cos x \sin 2 x^{\prime}(t)}{t^{4}}, \quad t>1 .
\end{gathered}
$$

We note that

$$
\begin{aligned}
& \frac{H\left(t, x^{\prime}(t), x(t)\right)}{g(x(t))} \\
& =\frac{x^{3} \cos x \sin 2 x^{\prime}(t)}{t^{4}} \times \frac{1}{x^{3}(t)} \leq \frac{1}{t^{4}}=p(t), \\
& \forall x^{\prime} \in \mathbb{R}, \quad x \in \mathbb{R}, \quad t \geq t_{0},
\end{aligned}
$$

hence,

$$
\begin{gathered}
r(t)=t>0, \\
8 \leq \psi(x(t))=8+\frac{x^{4}(t)}{x^{4}(t)+1}<9, \quad \forall x \in \mathbb{R}, \\
7 \leq \frac{f(y)}{y} 2=7+\frac{\left(x^{\prime}(t)\right)^{4}}{\left(x^{\prime}(t)\right)^{4}+1}<8, \quad \forall y \neq 0 .
\end{gathered}
$$

$$
\int_{T_{0}}^{t} \rho(s)[q(s)-p(s)] d s=\int_{T_{0}}^{t}\left(\frac{1}{s^{2}}-\frac{1}{s^{3}}\right) d s<\infty .
$$

Because $Z(s)=\rho(s)[q(s)-p(s)]-(1 / 4 A)\left(\left(\rho^{\prime}(s)\right)^{2} / \rho(s)\right) r(s)$ $=\left(1 / s^{2}\right)-\left(1 / s^{3}\right)-(1 / 4 A)$,

$$
\begin{gathered}
\lim _{t \rightarrow \infty} \inf \int_{T}^{t} Z(s) d s=\lim _{t \rightarrow \infty} \inf \int_{T}^{t}\left(\frac{1}{s^{2}}-\frac{1}{s^{3}}-\frac{1}{4 A}\right) d s=0, \\
\lim _{t \rightarrow \infty} \int_{T_{0}}^{t} \frac{1}{r(s) \rho(s)} \int_{s}^{\infty} Z(u) d u d s \\
=\lim _{t \rightarrow \infty} \int_{T_{0}}^{t} \frac{1}{s^{2}} \int_{s}^{\infty}\left(\frac{1}{u^{2}}-\frac{1}{u^{3}}-\frac{1}{4 A}\right) d u d s=\infty \\
\int_{\varepsilon}^{\infty} \frac{d y}{f(y)}=\int_{\varepsilon}^{\infty} \frac{d y}{y^{3}}=\left.\frac{-2}{y^{2}}\right|_{\varepsilon} ^{\infty}=\frac{2}{\varepsilon^{2}}<\infty \\
\int_{-\varepsilon}^{-\infty} \frac{d y}{f(y)}=\frac{2}{\varepsilon^{2}}<\infty
\end{gathered}
$$

Thus, from Theorem 7, it follows that the equation is oscillatory.

Remark 10. If we let $Z(t)=1$ and $f\left(x^{\prime}(t)\right)=x^{\prime}(t)$ in our Theorems 5 and 7, we will get Theorems 1 and 2 of Remili [7] which extends the results of Graef et al. [3]. 


\section{Conflict of Interests}

The authors declare that there is no conflict of interests regarding the publication of this paper.

\section{Acknowledgment}

This research has been completed with the support of these Grants: FRGS/1/2012/SG04/UKM/01/1, DIP-2012-31, and $\mathrm{FRGS} / 2 / 2013 / \mathrm{SG} 04 / \mathrm{UKM} / 02 / 3$.

\section{References}

[1] M. M. A. El-Sheikh, "Oscillation and nonoscillation criteria for second order nonlinear differential equations, I," Journal of Mathematical Analysis and Applications, vol. 179, no. 1, pp. 1427, 1993.

[2] S. R. Grace, "Oscillation theorems for nonlinear differential equations of second order," Journal of Mathematical Analysis and Applications, vol. 171, no. 1, pp. 220-241, 1992.

[3] J. R. Graef, S. M. Rankin, and P. W. Spikes, "Oscillation theorems for perturbed nonlinear differential equations," Journal of Mathematical Analysis and Applications, vol. 65, no. 2, pp. 375390, 1978.

[4] J. R. Graef and P. W. Spikes, "On the oscillatory behavior of solutions of second order nonlinear differential equations," Czechoslovak Mathematical Journal, vol. 36, no. 2, pp. 275-284, 1986.

[5] J. Ohriska and A. Zulova, "Oscillation criteria for second order nonlinear differential equation," IM Preprint A, vol. 10, pp. 1-11, 2004.

[6] C. G. Philos and I. K. Purnaras, "Oscillations in superlinear differential equations of second order," Journal of Mathematical Analysis and Applications, vol. 165, no. 1, pp. 1-11, 1992.

[7] M. Remili, "Oscillation theorem for perturbed nonlinear differential equations," International Mathematical Forum, vol. 3, no. 11, pp. 513-524, 2008.

[8] M. Remili, "Oscillation criteria for second order nonlinear perturbed differential equations," Electronic Journal of Qualitative Theory of Differential Equations, no. 25, pp. 1-11, 2010.

[9] P. Temtek and A. Tiryaki, "Oscillation criteria for a certain second-order nonlinear perturbed differential equations," Journal of Inequalities and Applications, vol. 524, pp. 1-12, 2013.

[10] J. V. Manojlovic, "Oscillation criteria for second-order sublinear differential equation," Computers and Mathematics with Applications, vol. 39, no. 9, pp. 161-172, 2000.

[11] M. Kirane and Y. V. Rogovchenko, "On oscillation of nonlinear second order differential equation with damping term," Applied Mathematics and Computation, vol. 117, no. 2-3, pp. 177-192, 2001.

[12] A. Tiryaki and B. Ayanlar, "Oscillation theorems for certain nonlinear differential equations of second order," Computers and Mathematics with Applications, vol. 47, no. 1, pp. 149-159, 2004.

[13] W.-T. Li, "Oscillation of certain second-order nonlinear differential equations," Journal of Mathematical Analysis and Applications, vol. 217, no. 1, pp. 1-14, 1998.

[14] C. G. Philos, “On a Kamenev's integral criterion for oscillation of linear differential equations of second order," Utilitas Mathematica, vol. 24, pp. 277-289, 1983.
[15] J. V. Manojlovic, "Integral averages and oscillation of secondorder nonlinear differential equations," Computers and Mathematics with Applications, vol. 41, no. 12, pp. 1521-1534, 2001.

[16] C. G. Philos, "Oscillation of sublinear differential equations of second order," Nonlinear Analysis, vol. 7, no. 10, pp. 1071-1080, 1983.

[17] C. G. Philos, "Integral averages and oscillation of second order sublinear differential equations," Differential and Integral Equations, vol. 4, no. 1, pp. 205-213, 1991. 


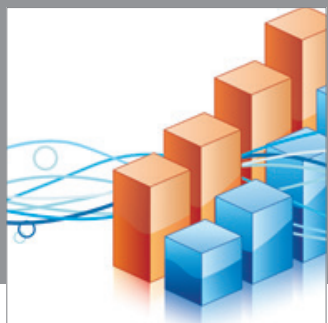

Advances in

Operations Research

mansans

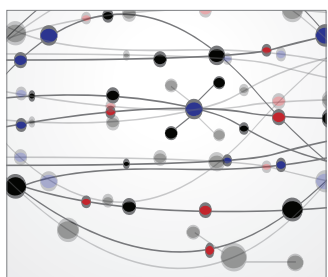

The Scientific World Journal
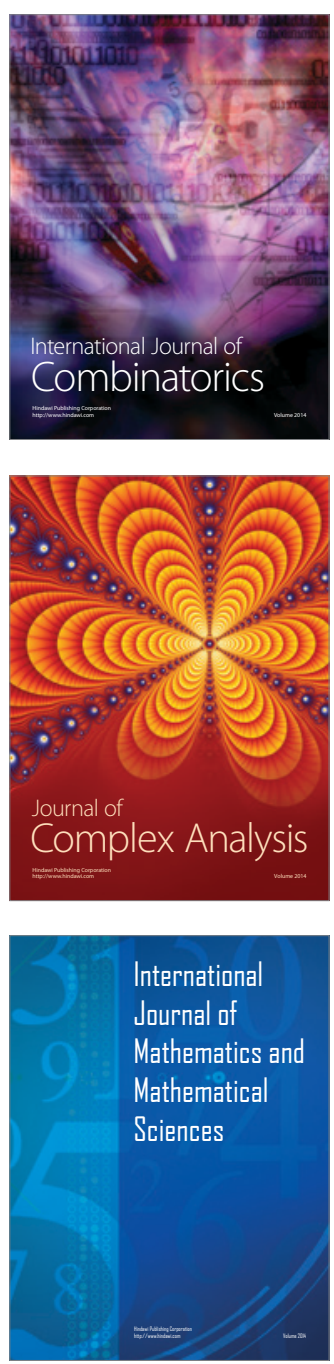
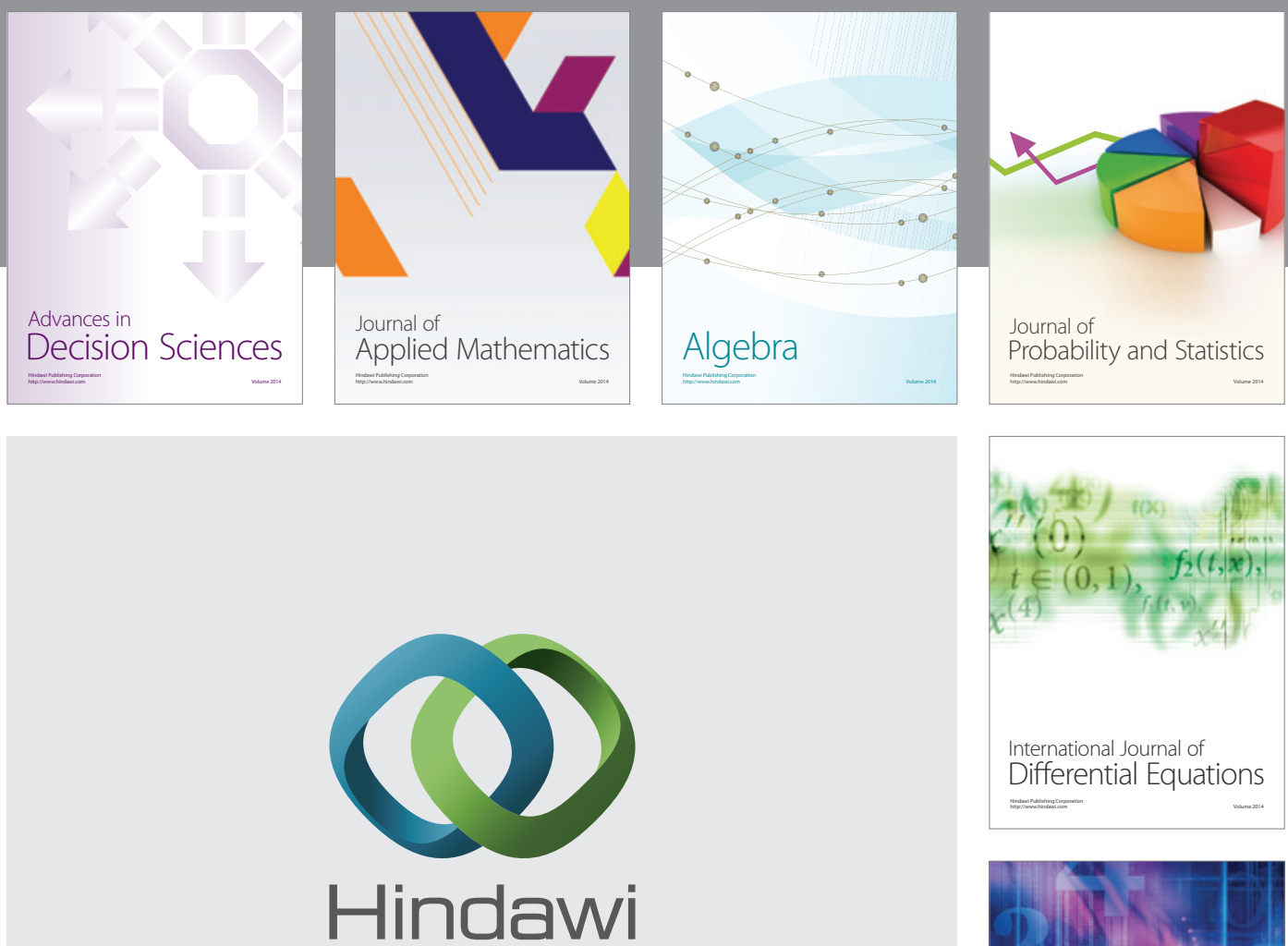

Submit your manuscripts at http://www.hindawi.com
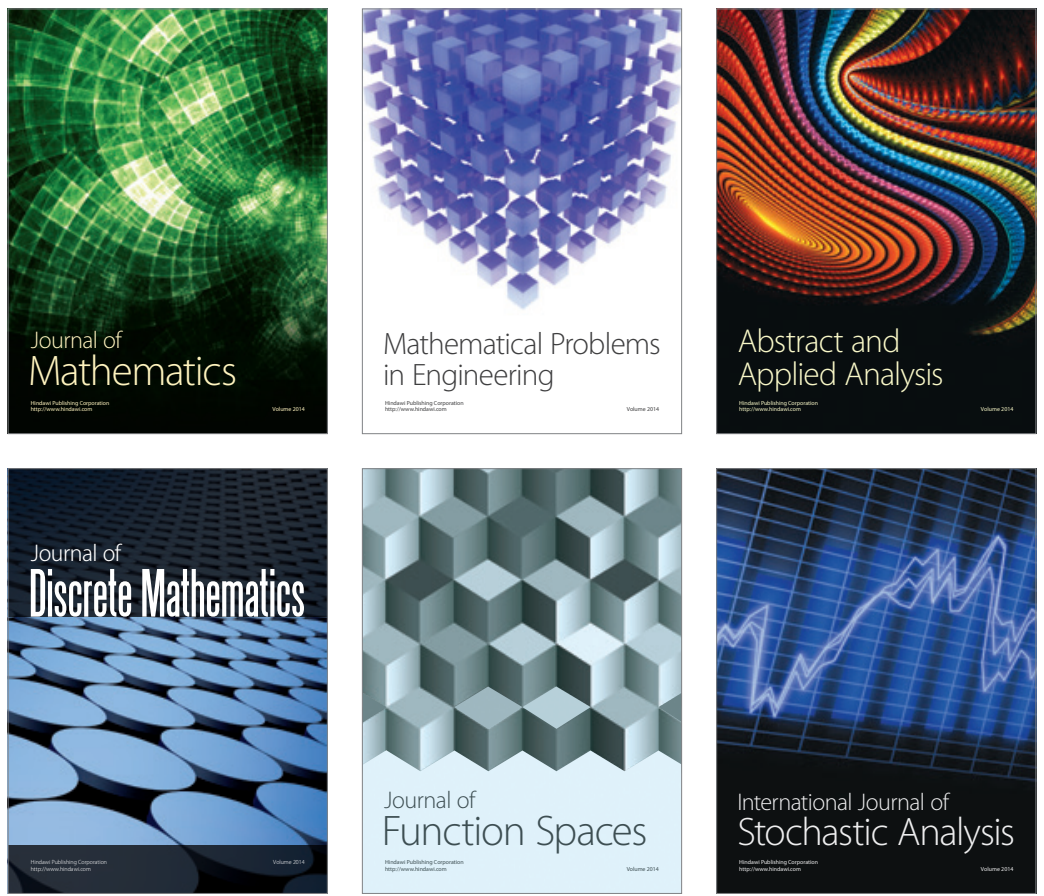

Journal of

Function Spaces

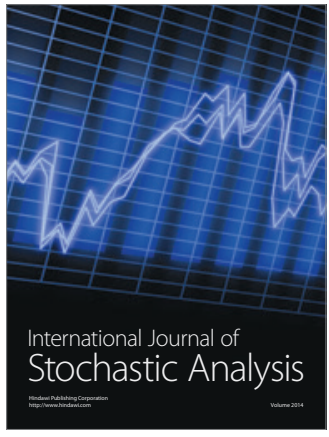

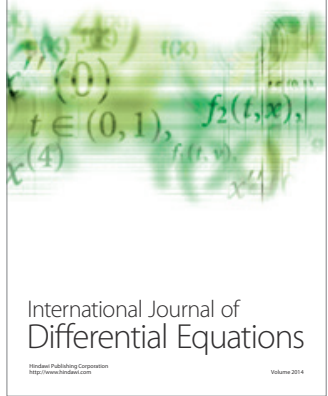
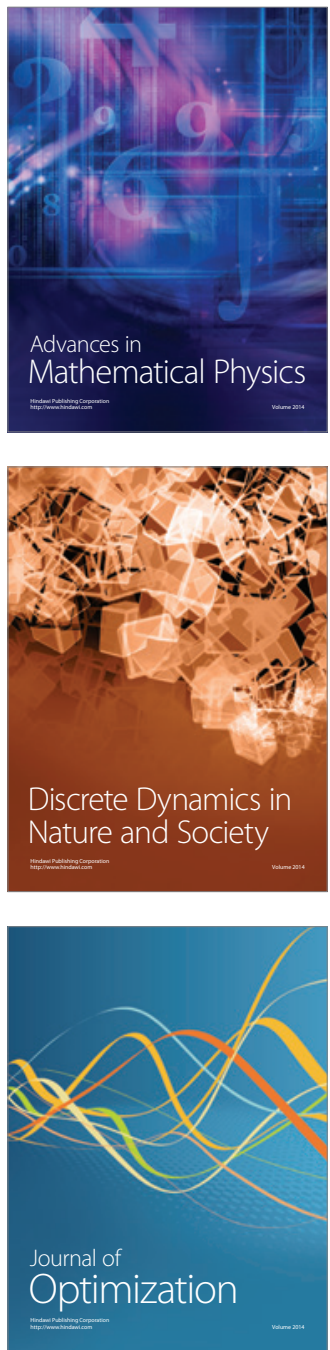